\title{
Managing hyperglycemia in patients with Cushing's disease treated with pasireotide: medical expert recommendations
}

\author{
Annamaria Colao · Christophe De Block • \\ Maria Sonia Gaztambide · Sudhesh Kumar • \\ Jochen Seufert • Felipe F. Casanueva
}

Published online: 7 April 2013

(C) The Author(s) 2013. This article is published with open access at Springerlink.com

\begin{abstract}
To recommend an approach to monitoring and treating hyperglycemia in pasireotide-treated patients with Cushing's disease, a severe clinical condition caused by a pituitary adenoma hypersecreting adrenocorticotropic hormone. Advisory Board meeting of ten European experts in pituitary disease and diabetes mellitus in Munich, Germany, on February 23, 2012, to obtain expert recommendations. Cushing's disease presents a number of management challenges. Pasireotide, a novel agent for the treatment of Cushing's disease with proven biochemical and clinical efficacy, improves outcomes and expands treatment options. Clinical trials have shown that the pasireotide adverse event profile is similar to that of other somatostatin analogs, except for a higher frequency of hyperglycemia. Mechanistic studies in healthy volunteers suggest that pasireotide-associated hyperglycemia is due to reduced secretion of glucagon-like peptide (GLP)-1, glucosedependent insulinotropic polypeptide, and insulin; however, it is associated with intact postprandial glucagon secretion. Individual patients' results demonstrate effective hyperglycemia management by following standard guidelines for the
\end{abstract}

\section{A. Colao $(\bowtie)$}

Dipartimento di Medicina Clinica e Chirurgia,

Università di Napoli Federico II, Naples, Italy

e-mail: colao@unina.it

C. De Block

Department of Endocrinology, Diabetology and Metabolism, Antwerp University Hospital, Edegem, Belgium

\section{S. Gaztambide}

Department of Endocrinology, University Hospital Cruces

(UPV-EHU), Vizcaya, Spain

M. S. Gaztambide

CIBERDEM (Centro de Investigación Biomédica en Red de

Diabetes y Enfermedades Metabólicas Asociadas; ISCIII),

Madrid, Spain treatment of diabetes mellitus with individual adaptation to the specific underlying pathophysiology, i.e., preferential use of GLP-1 based-medications. Patients on pasireotide treatment should be monitored for changes in glucose metabolism and hyperglycemia. Diabetes mellitus should be managed by initiation of medical therapy with metformin and staged treatment intensification with a dipeptidyl peptidase-4 inhibitor, with a switch to a GLP-1 receptor agonist and initiation of insulin, as required, to achieve and maintain glycemic control. Further research into hyperglycemia following pasireotide treatment will help refine the optimal strategy in Cushing's disease.

Keywords Cushing's disease · Glucagon-like-peptide-1 · Hyperglycemia $\cdot$ Pasireotide $\cdot$ Recommendations

\section{Introduction}

Cushing's disease is a severe clinical condition caused by a pituitary adenoma hypersecreting adrenocorticotropic

\author{
S. Kumar \\ University Hospital, Coventry, UK \\ J. Seufert \\ Division of Endocrinology and Diabetology, Department of \\ Internal Medicine II, University Hospital of Freiburg, \\ Freiburg, Germany \\ F. F. Casanueva \\ Department of Medicine, Santiago de Compostela University, \\ Santiago de Compostela, Spain \\ F. F. Casanueva \\ CIBER Obesity and Nutrition, ISCIII, Madrid, Spain
}


hormone (ACTH). The persistently high levels of ACTH lead to chronic hypersecretion of cortisol by the adrenal glands, which negatively affects many tissues and organs in the body. Cushing's disease accounts for $>70 \%$ of the causes of endogenous chronic hypercortisolism, other causes include ectopic ACTH secretion by tumors, adrenal adenoma or carcinoma, macronodular or micronodular adrenal hyperplasia, and, more rarely, primary pigmented nodular adrenocortical disease and McCune-Albright syndrome [1]. Cushing's disease is a rare disorder associated with substantial morbidity and mortality. It has an annual incidence of 1.2-2.4 cases per million population $[2,3]$ and a prevalence of 29.1 per million [3]. The effects of Cushing's disease include metabolic and cardiovascular complications, osteoporosis and other bone alterations, kidney stones, autoimmune diseases, and susceptibility to opportunistic infections [4-15]. Mortality in patients with active Cushing's disease is 4-times higher than in age- and sex-matched controls [3, 16].

The goals for treating Cushing's disease are the reversal or amelioration of clinical symptoms by the normalization of cortisol levels to achieve minimal morbidity while incurring the fewest side effects possible. This can be realized by the removal of the tumor mass (while preserving pituitary function) and inhibition of tumor growth to achieve longterm control without recurrence. Several interventions exist to treat Cushing's disease, including surgery, radiotherapy, and medical therapy. Although initial surgery is most frequently used as the first-line option, it often fails [1, 17]. Radiotherapy may be an option when initial surgery is unsuccessful. However, therapeutic effects are often delayed and become apparent sometimes only after many years [18]. Also, radiotherapy may have side effects including partial or complete pituitary deficiency [19]. Medical therapies can be useful in patients with persistent disease or recurrence after surgery, but many of the drugs applied have limited effects or are not approved for Cushing's disease [17,20]. Adrenostatic medical therapies, including ketoconazole, metyrapone, mitotane, and etomidate, may also be used for Cushing's disease $[17,20]$. Novel therapies directed to reduce cortisol levels or cortisol biological action such as 11-beta-hydroxylase inhibitors or blockers of the glucocorticoid receptor are also in development [21, 22].

Pasireotide (Signifor ${ }^{\circledR}$; Novartis Pharma AG, Basel, Switzerland) is the first medical therapy approved in the European Union to treat adult patients with Cushing's disease for whom surgery is not an option or for whom surgery has failed. Pasireotide is the only medical treatment targeting the pituitary adenoma and has demonstrated long-term effectiveness for the biochemical control and clinical improvement of patients with Cushing's disease $[23,24]$. It was approved based on the results of a pivotal phase III study [23]. In this study, $26.3 \%$ of patients treated with pasireotide $900 \mu \mathrm{g}$ twice daily and $14.6 \%$ treated with pasireotide $600 \mu \mathrm{g}$ twice daily achieved normal urinary free cortisol (UFC) levels at month 6 without dose escalation, and 50 of 103 patients had a substantial reduction (either normalization or at least reduction from baseline) in the UFC levels at 6 months [23]. In patients treated with pasireotide $900 \mu \mathrm{g}$ twice daily and who had baseline UFC levels between 1.5- and 2-times the upper limit of normal, half achieved normalization of UFC [23]. Clinical signs and symptoms of Cushing's disease improved in most patients in this study, including a significant reduction in body weight, systolic- and diastolicblood pressure, and low-density lipoprotein cholesterol levels, with a reduction in mean UFC [23].

\section{Hyperglycemia secondary to hypercortisolism}

Patients with Cushing's disease are at a high risk of developing impaired glucose tolerance (IGT) and manifest diabetes secondary to hypercortisolism. A number of pathophysiological mechanisms underlie glucocorticoid-induced diabetes development in patients with Cushing's disease (Fig. 1) [25, 26]. In general, chronic hypercortisolism blocks or impedes the action of insulin on peripheral tissues, such as liver, muscle and adipose tissue, leading to increased insulin resistance, and it partially inhibits insulin release by the pancreatic beta-cells. In particular, the glucocorticoid excess of patients with Cushing's disease has a number of effects on glucose metabolism in hepatic tissue. These effects may be direct, such as stimulating gluconeogenesis through the induction of expression of essential enzymes [e.g., glucose transporter type 4 , protein phosphatase 1 , glycogen synthase kinase 3 , and glycogen synthase (Fig. 1 provides an overview of the pathophysiological mechanisms of glucocorticoid-induced diabetes)] stimulating lipolysis and proteolysis with production of free fatty acids and amino acids which are substrates for gluconeogenesis, or the potentiation of hormone actions (especially glucagon) involved in glucose metabolism. Or they may be indirect, through the inhibition of insulin sensitivity by the depletion of glycogen storage in the liver [27-30]. The cortisol excess in patients with Cushing's disease also leads to increased appetite and other central effects. Moreover, glucocorticoids induce pancreatic beta-cell dysfunction by inhibiting several signaling pathways and interfering at several steps in the insulin signaling cascade, particularly those that involve glucose cycling, glucose 6 phosphate, and protein kinase $\mathrm{A}$ and $\mathrm{C}$ activation (Fig. 1) [25, 26], all which may contribute to the risk of hyperglycemia secondary to hypercortisolism. Over time, patients are therefore likely to develop a disturbance of glucose homeostasis. In a recent study, the prevalence of IGT was $27 \%$ in patients with Cushing's disease and adrenal adenomas compared with $10 \%$ in matched controls $(P<0.001)$ [31], which may develop further to 
manifest diabetes mellitus induced by the endocrine disease [32]. According to American Diabetes Association (ADA) classification, this type of diabetes can be classified under "Other specific types of diabetes due to other causes" or type 3 diabetes mellitus [32]. This diabetes should be managed in a similar way to type 2 diabetes mellitus (T2DM).

Generally, 40-45\% of patients with Cushing's disease have been reported to develop diabetes and a further 10-30\% of patients have IGT [25, 33]. This compares with a phase II study which found that $5 \%$ of patients with acromegaly treated with pasireotide developed diabetes, in addition to $7 \%$ of patients showing increased blood glucose levels, and $5 \%$ increased glycated hemoglobin $\left(\mathrm{HbA}_{1 \mathrm{C}}\right)$ levels [34]. However, these figures may be an under-estimation of the true number of patients affected as specific testing of diabetes mellitus is not always performed in these patients.

\section{Hyperglycemia secondary to pasireotide treatment}

Although the general safety profile of pasireotide in the pivotal study was consistent with first-generation somatostatin analogs (octreotide and lanreotide), hyperglycemia was observed in the majority of patients, with hyperglycemia-related adverse events reported in $72.8 \%$ of patients
[23]. Adverse events such as hyperglycemia and diabetes mellitus classified as grade 3 and 4 (according to the National Cancer Institute Common Terminology for Adverse Events version 3.0 [35]) occurred in up to $20 \%$ of patients [23]. Levels of glucose and $\mathrm{HbA}_{1 \mathrm{C}}$ increased soon after the initiation of pasireotide treatment, and remained elevated throughout the treatment period in this study.

The pathophysiology of hyperglycemia secondary to pasireotide treatment, and methods for its management were investigated in two mechanistic studies in healthy volunteers [36]. These investigations have demonstrated that pasireotide administered over a short 8-day period decreases secretion of insulin by the beta-cell and intestinal secretion of glucagon-like peptide (GLP)-1 and glucose-dependent insulinotropic peptide, possibly through somatostatin receptor mediated effects on pancreatic beta-cells and entero-endocrine cells [36]. Hepatic and peripheral insulin sensitivity remains unchanged [36]. This diminished insulinotropic incretin effect may further contribute to the reduced insulin secretion that was observed. The studies suggest that in healthy volunteers dipeptidyl peptidase (DPP)-4 inhibitors (e.g., sitagliptin, vildagliptin, saxagliptin, linagliptin) and GLP-1 receptor agonists (e.g., liraglutide, exenatide) may be the most effective agents for reducing pasireotide-associated hyperglycemia [36].

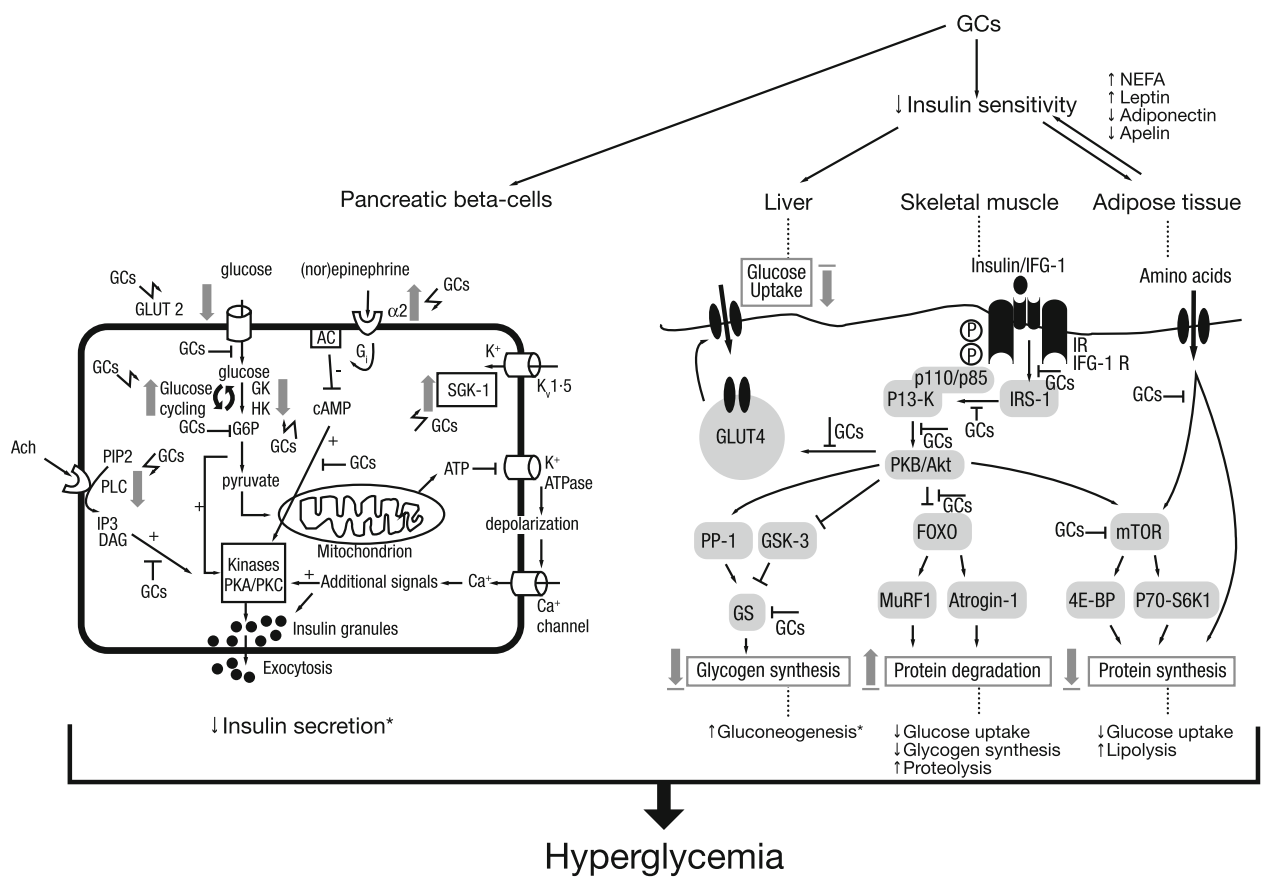

Fig. 1 Pathophysiological mechanisms of glucocorticoid-induced diabetes. Adapted with permission from Refs. [25] (c) 2011 Elsevier; and [26] (C) 2009 John Wiley and Sons. AC adenyl cyclase, Ach acetylcholine, ATP adenosine triphosphate, $c A M P$ cyclic adenosine monophosphate, $D A G$ diacylglycerol, $4 E-B P 1$ eIF4E-binding protein 1, $G 6 P$ glucose-6-phosphatase, $G i$ G-coupled inhibitory protein, $G C$ glucocorticoid, $G K$ glucokinase, GLUT2 glucose transporter 2, GLUT4 glucose transporter 4, GS glycogen synthase, GSK-3 glycogen synthase kinase-3, $H K$ hexokinase, $I F G$ - 1 insulin-like growth factor$1, I P 3$ inositol triphosphate, $K v 1.5$ voltage-dependent $\mathrm{K}$ channel, $I R$ insulin receptor, IRS-1 insulin receptor substrate-1, mTOR mammalian target of rapamycin, $M u R F-1$ muscle ring finger-1, PI3-K phosphatidylinositol-3 kinase, $P I P 2$ phosphatidylinositol biphosphate, $P K A$ protein kinase A, $P K B$ protein kinase $\mathrm{B}, P K C$ protein kinase $\mathrm{C}$, $P L C$ phospholipase C, $P P-1$ protein phosphatase-1, $S G K-1$ serumand glucocorticoid inducible kinase-1, $S 6 K 1$ protein S6 kinase 1 
On February 23, 2012 a group of ten European experts in the field of pituitary diseases and diabetes mellitus met at an Advisory Board meeting in Munich, Germany, to propose an approach to the management of hyperglycemia in patients with Cushing's disease treated with pasireotide. This paper provides medical expert recommendations on the monitoring and treatment of hyperglycemia in patients with Cushing's disease. The recent approval of pasireotide as a medical therapy for patients with persistent or recurrent Cushing's disease for whom surgery is not an option or for whom surgery has failed renders these recommendations especially timely.

\section{Methods}

These recommendations were prepared from the outcomes of the Advisory Board meeting and this article presents a summary of the discussions of the experts.

\section{Results}

\section{Expert recommendations}

\section{For monitoring patients with Cushing's disease treated with pasireotide}

The results of the pivotal phase III study of pasireotide in patients with Cushing's disease demonstrated that the majority of patients treated with pasireotide will develop hyperglycemia [23]. Therefore, all patients treated with pasireotide should be monitored for the development of IGT or manifest diabetes mellitus. Current definitions used by the ADA for the diagnosis of manifest diabetes are an $\mathrm{HbA}_{1 \mathrm{C}}$ level of $\geq 6.5 \%$, a fasting plasma glucose level of $\geq 126 \mathrm{mg} / \mathrm{dL}(7.0 \mathrm{mmol} / \mathrm{L})$, a 2 -h plasma glucose level of $\geq 200 \mathrm{mg} / \mathrm{dL}(11.1 \mathrm{mmol} / \mathrm{L})$ during an oral glucose tolerance test; or in patients with classic symptoms of hyperglycemia or hyperglycemic crisis, a random plasma glucose level of $\geq 200 \mathrm{mg} / \mathrm{dL}$ (11.1 mmol/L) [32].

Patients with normal glucose metabolism prior to pasireotide therapy should self-monitor their fasting and postprandial glucose levels by taking several blood glucose measurements per day (e.g., before and $2 \mathrm{~h}$ after breakfast, lunch, and dinner). They should do so twice in the first week and once weekly thereafter. Patients with impaired fasting glycemia (IFG) and/or IGT, and patients with diabetes under treatment, must be closely monitored on a daily basis at the start of treatment with pasireotide and, if needed, treatment shall be adjusted or changed in order to control the new level of glycemia. Their treating physician should follow-up closely (e.g., after 1, 2, and 4 weeks) to assess the emergence of any hyperglycemia and initiate appropriate therapy.

\section{For treating hyperglycemia secondary to pasireotide therapy in patients with Cushing's disease}

Treatment for patients with Cushing's disease, whether they present with IFG and/or IGT or diabetes, or develop
Fig. 2 Recommendations on the monitoring and treatment of hyperglycemia in patients with Cushing's disease treated with pasireotide. In case patients fail to control glucose levels with GLP-1 agonists, as stated in the text, we suggest moving to insulin. DDP-4 dipeptidyl peptidase-4, $D M$ diabetes mellitus, $G L P-1$ glucagon-like peptide-1, $H b A_{I C}$ glycated hemoglobin, $I F G$ impaired fasting glycemia, $I G T$ impaired glucose tolerance, $N G T$ normal glucose tolerance, $O A D$ oral antidiabetic drugs, $S M B G$ selfmonitoring of blood glucose

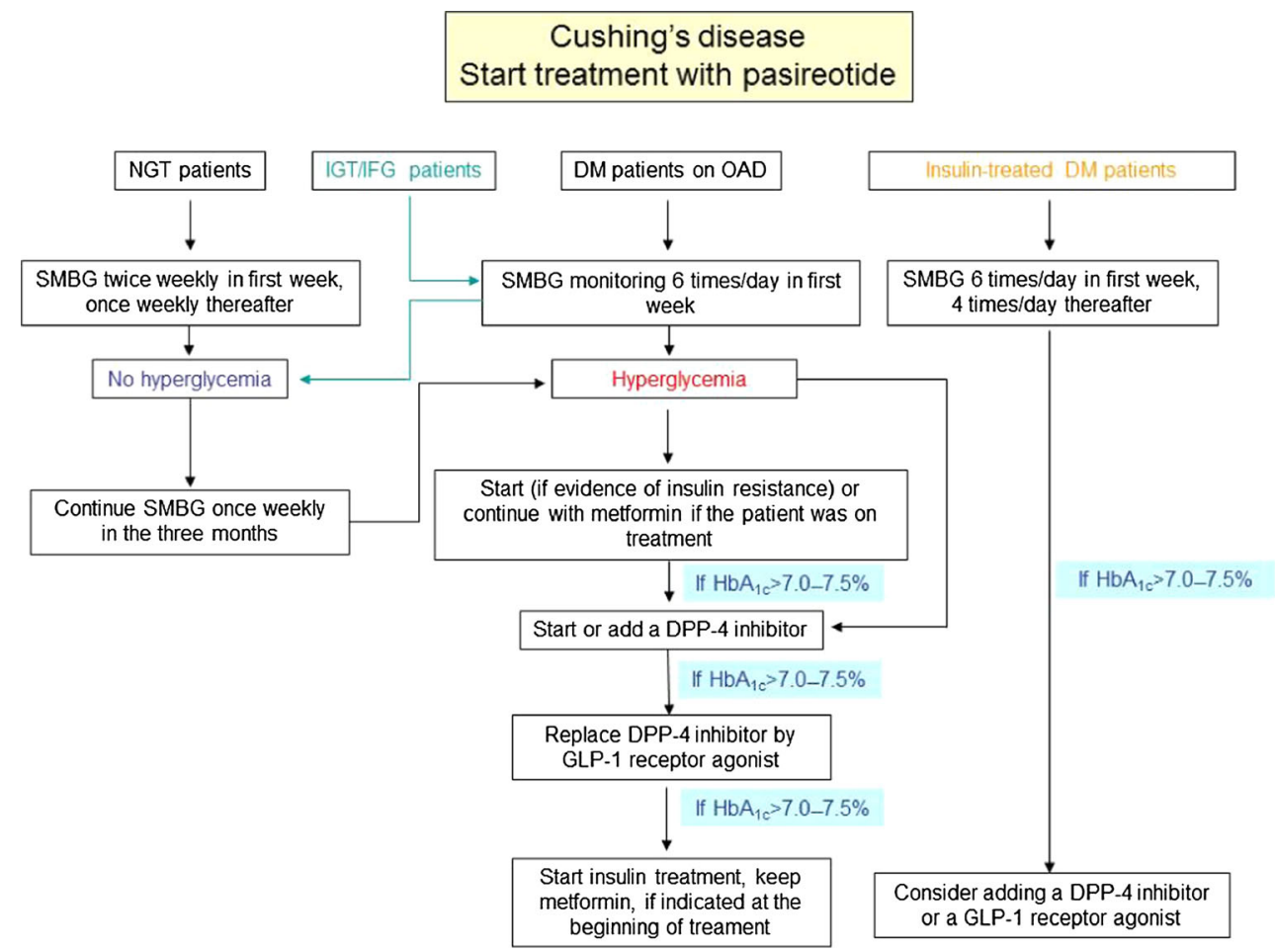


hyperglycemia secondary to treatment with pasireotide, should be based on the currently recommended treatment algorithms for T2DM. Thus, individually tailored glycemic targets of $\mathrm{HbA}_{1 \mathrm{C}}$ level of $<7.0-7.5 \%(<53-58 \mathrm{mmol} / \mathrm{mol})$ are appropriate unless the treating clinician perceives a risk due to hypoglycemia, or if the patient is unlikely to survive long enough to be at risk of complications. Medical treatment should include dietary modification, exercise, and education. In patients with evidence of insulin resistance, medication should be initiated with metformin as the firstline therapy unless contraindicated or not well tolerated (Fig. 2) $[33,37]$.

As pasireotide-related hyperglycemia is associated with both decreased insulin secretion and a reduced incretin response [34], anti-hyperglycemic treatment in patients with Cushing's disease treated with pasireotide should preferentially address these two pathophysiological mechanisms. If control of glycemia is not achieved or maintained with metformin alone, combination therapy with agents targeting the incretin-pathway is recommended [36]. Based on the preliminary data mentioned above [36], it is suggested that in hyperglycemia secondary to pasireotide treatment, metformin in combination with a GLP-1-based treatment option may be specifically advantageous (Fig. 2). Therefore, in a first step combination, therapy with a DPP4 inhibitor may be established. In addition, to target the specific pathophysiology of hyperglycemia secondary to pasireotide treatment, this combination provides the specific advantages of a low hypoglycemic potential together with a neutral effect on body weight. Although combination therapies of metformin with sulfonylureas and/or pioglitazone may also be possible theoretically, these are not recommended as sulphonylureas stimulate insulin secretion in a glucose-independent manner and, therefore, are associated with a substantial risk of hypoglycemia and weight gain. Furthermore, treatment failure due to a decline of pancreatic beta-cell function may occur more rapidly with sulphonylurea therapy. Pioglitazone primarily targets insulin resistance and does not, therefore, represent a pathophysiologically-oriented treatment option for hyperglycemia secondary to pasireotide therapy. Moreover, pioglitazone treatment has been associated with an increase in body weight, fluid retention, and bone fractures-all side effects that are most unwanted in Cushing's disease patients [38].

If glycemic targets are not reached with the metformin plus DDP-4 inhibitor combination, the DPP-4 inhibitor may be replaced by a GLP-1 receptor agonist. These GLP1 analogs have demonstrated an $\mathrm{HbA}_{1 \mathrm{C}}$-lowering effect that is superior to that of DPP-4 inhibitors without increase of hypoglycemia risk. In addition, they provide the potential to reduce body weight, which is desirable in Cushing's disease. If hyperglycemia secondary to pasireotide treatment in patients with Cushing's disease remains uncontrolled by these combinations, establishing insulin therapy together with maintained metformin treatment may be necessary. In these cases, initial combination therapy of metformin with a once-daily application of a long-acting basal insulin analog (either glargine or detemir since they have similar efficacy on glucose target [39]) targeting levels of fasting plasma glucose considered acceptable by the treating physician, may be the first option. If the individual $\mathrm{HbA}_{1 \mathrm{C}}$ targets are not met or the postprandial glucose levels are high with this basal insulinsupported anti-diabetic therapy, then prandial insulin therapy also has to be finally established.

These recommendations are supported by similar proposals on the management of hyperglycemia in Cushing's disease developed from a review of the existing literature [40]. Reznik et al. suggest that patients should be tested for impaired glucose regulation before initiating treatment with pasireotide. They should be monitored for glycemic control on initiation of pasireotide and also as part of regular follow-ups and, if glycemic control deteriorates during pasireotide therapy, they should be treated with antidiabetic therapy as appropriate [40].

\section{Further research}

A study of patients with Cushing's disease who perform regular monitoring of blood glucose would provide information to help stratify patients by risk of hyperglycemia and assist the development of specific guidelines for managing this risk in the future. At this point, however, it seems prudent to apply a similar clinical risk estimation for the development of glycemic disorders in patients with Cushing's disease as is standard in patients at-risk for classical T2DM. This involves assessing features of the metabolic syndrome, such as visceral obesity, concomitant dyslipoproteinemia and hypertension, and familial background for metabolic disease. Whether validated diabetes risk questionnaires such as the ones developed from the Study to Prevent Non-Insulin Dependent Diabetes Mellitus (STOP-NIDDM) and the Finnish Cardiovascular Risk Factor (FINDRISK) [41, 42], may help to identify patients at risk in the setting of Cushing's disease is unknown.

Significant progress in understanding the fundamental pathophysiology of hyperglycemia secondary to pasireotide treatment has been made since the phenomenon was first recognized. However, further research on how pasireotide influences insulin biosynthesis and secretion, and insulin sensitivity in patients with Cushing's disease will help to define the optimum treatment strategies for hyperglycemia in this difficult to manage condition. 
Although metformin followed by the addition of a DPP4 inhibitor, with a switch from the DPP-4 inhibitor to a GLP-1 receptor agonist or insulin therapy as required to maintain glycemic targets, is suggested as a suitable treatment of hyperglycemia secondary to pasireotide therapy, the efficacy of anti-hyperglycemic regimens needs to be further explored in a clinical trial in patients with Cushing's disease.

\section{Summary}

Patients with Cushing's disease have a high risk of hyperglycemia due to the long-term effect of hypercortisolism, and emergence or worsening of hyperglycemia is frequently observed secondary to pasireotide treatment. However, mechanistic studies and individual observations in patients with Cushing's disease suggest that pasireotiderelated hyperglycemia responds to anti-diabetic agents following similar protocols recommended for the treatment of T2DM, with a specific focus on GLP-1-based medications. More research is necessary to confirm these results and to refine the recommendations for diagnosis and treatment of hyperglycemia in patients with Cushing's disease in order to optimize their management.

\begin{abstract}
Acknowledgments The authors would like to thank all the medical experts who participated in the Advisory Board meeting: Marco Boscaro (University Politecnica delle Marche, Ancona, Italy), Diego Ferone (Department of Endocrinology and Medical Sciences [DISEM], University of Genoa, Genoa, Italy), Sten Madsbad (Department of Endocrinology, Hvidovre University Hospital, University of Copenhagen, Hvidovre, Denmark), and Bruno Vergès (Service Endocrinologie et Maladies Métaboliques, Hôpital du Bocage, CHU Dijon, Dijon, France). The authors received writing/ editorial support in the preparation of this manuscript by Excerpta Medica, funded by Novartis Oncology Region Europe.
\end{abstract}

Conflict of interest The Advisory Board expert meeting was funded by Novartis Oncology Region Europe. Dr. Colao has received speaker honoraria and/or consulting fees from Ipsen, Lilly, Novartis, Pfizer. Dr. De Block has served on advisory panels for Abbott, Menarini Diagnostics, Eli Lilly and Company, NovoNordisk, and Novartis; has received honoraria as a member of the speaker's bureau of Abbott, Menarini Diagnostics, Eli Lilly and Company, and Sanofi. Dr. Gaztambide has received speaker honoraria and/or consulting fees from AstraZeneca/Bristol-Myers Squibb, Boehringer Ingelheim/Lilly, Novartis, NovoNordisk, and Pfizer. Dr. Kumar has received grant support and lecture fees from NovoNordisk. Dr. Seufert has received speaker honoraria and/or consulting fees from Takeda, Bayer, Novartis, Merck Sharp Dohme (MSD), AstraZeneca/Bristol-Myers Squibb, NovoNordisk, Sanofi, Berlin Chemie, Lilly, Merck, Roche, Ipsen, Pfizer, Janssen, and Lifescan. Dr. Casanueva has received honoraria and/or consulting fees from Pfizer, NovoNordisk, Novartis, and Boehringer Ingelheim.

Open Access This article is distributed under the terms of the Creative Commons Attribution License which permits any use, distribution, and reproduction in any medium, provided the original author(s) and the source are credited.

\section{References}

1. Newell-Price J, Bertagna X, Grossman AB, Nieman LK (2006) Cushing's syndrome. Lancet 367:1605-1617

2. Lindholm J, Juul S, Jørgensen JO et al (2001) Incidence and late prognosis of Cushing's syndrome: a population-based study. J Clin Endocrinol Metab 86:117-123

3. Etxabe J, Vazquez JA (1994) Morbidity and mortality in Cushing's disease: an epidemiological approach. Clin Endocrinol (Oxf) 40:479-484

4. Chanson P, Salenave S (2010) Metabolic syndrome in Cushing's syndrome. Neuroendocrinology 92(Suppl 1):96-101

5. Colao A, Pivonello R, Spiezia S et al (1994) Persistence of increased cardiovascular risk in patients with Cushing's disease after five years of successful cure. J Clin Endocrinol Metab 84:2664-2672

6. Faggiano A, Pivonello R, Filippella M et al (2001) Spine abnormalities and damage in patients cured from Cushing's disease. Pituitary 4:153-161

7. Faggiano A, Pivonello R, Ruosi C et al (2001) Uncommon clinical course of multiple osteochondromatosis in a patient with a long-term history of Cushing's disease. Pituitary 4:187-193

8. Di Somma C, Pivonello R, Loche S et al (2002) Severe impairment of bone mass and turnover in Cushing's disease: comparison between childhood-onset and adulthood-onset disease. Clin Endocrinol (Oxf) 56:153-158

9. Faggiano A, Pivonello R, Melis D et al (2002) Evaluation of circulating levels and renal clearance of natural amino acids in patients with Cushing's disease. J Endocrinol Invest 25:142151

10. Faggiano A, Pivonello R, Spiezia S et al (2003) Cardiovascular risk factors and common carotid artery caliber and stiffness in patients with Cushing's disease during active disease and 1 year after disease remission. J Clin Endocrinol Metab 88:2527-2533

11. Faggiano A, Pivonello R, Melis D et al (2003) Nephrolithiasis in Cushing's disease: prevalence, etiopathogenesis, and modification after disease cure. J Clin Endocrinol Metab 88:2076-2080

12. Pivonello R, De Leo M, Vitale P et al (2010) Pathophysiology of diabetes mellitus in Cushing's syndrome. Neuroendocrinology 92(Suppl 1):77-81

13. van der Eerden AW, den Heijer M, Oyen WJ, Hermus AR (2007) Cushing's syndrome and bone mineral density: lowest $\mathrm{Z}$ scores in young patients. Neth J Med 65:137-141

14. Bakker RC, Gallas PR, Romijn JA, Wiersinga WM (1998) Cushing's syndrome complicated by multiple opportunistic infections. J Endocrinol Invest 21:329-333

15. Colao A, Pivonello R, Faggiano A et al (2000) Increased prevalence of thyroid autoimmunity in patients successfully treated for Cushing's disease. Clin Endocrinol (Oxf) 53:13-19

16. Graversen D, Vestergaard P, Stochholm K, Gravholt CH, Jørgensen JO (2012) Mortality in Cushing's syndrome: a systematic review and meta-analysis. Eur J Intern Med 23:278-282

17. Biller BM, Grossman AB, Stewart PM et al (2008) Treatment of adrenocorticotropin-dependent Cushing's syndrome: a consensus statement. J Clin Endocrinol Metab 93:2454-2462

18. Castinetti F, Nagai M, Dufour H et al (2007) Gamma knife radiosurgery is a successful adjunctive treatment in Cushing's disease. Eur J Endocrinol 156:91-98

19. Devin JK, Allen GS, Cmelak AJ, Duggan DM, Blevins LS (2004) The efficacy of linear accelerator radiosurgery in the management of patients with Cushing's disease. Stereotact Funct Neurosurg 82:254-262

20. Dang CN, Trainer P (2007) Pharmacological management of Cushing's syndrome: an update. Arq Bras Endocrinol Metabol 51:1339-1348 
21. Bertagna X, Pivonello R, Fleseriu M, et al (2012) Patients with Cushing's disease achieve normal urinary cortisol with LCI699, a

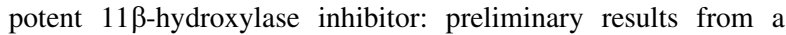
multicenter, proof-of-concept study. In: 15th International and 14th European Congress of Endocrinology (ICE/ECE 2012), Florence, Italy (abstract OC1.2)

22. Fleseriu M, Biller BMK, Findling JW et al (2012) Mifepristone, a glucocorticoid receptor antagonist, produces clinical and metabolic benefits in patients with Cushing's syndrome. J Clin Endocrinol Metab 97:2039-2049

23. Colao A, Petersenn S, Newell-Price J et al (2012) A 12-month phase 3 study of pasireotide in Cushing's disease. N Engl J Med 366:914-924

24. Bertherat J, Ludlam WH, Pivonello R, et al (2012) Long-term use of pasireotide in Cushing's disease: 24-month safety results from a randomized phase III study. In: 15th International and 14th European Congress of Endocrinology (ICE/ECE 2012), Florence, Italy (abstract P1405)

25. Mazziotti G, Gazzaruso C, Giustina A (2011) Diabetes in Cushing syndrome: basic and clinical aspects. Trends Endocrinol Metab 22:499-506

26. van Raalte DH, Ouwens DM, Diamant M (2009) Novel insights into glucocorticoid-mediated diabetogenic effects: towards expansion of therapeutic options? Eur J Clin Invest 39:81-93

27. Pivonello R, Faggiano A, Lombardi G, Colao A (2005) The metabolic syndrome and cardiovascular risk in Cushing's syndrome. Endocrinol Metab Clin North Am 34:327-339

28. Resmini E, Minuto F, Colao A, Ferone D (2009) Secondary diabetes associated with principal endocrinopathies: the impact of new treatment modalities. Acta Diabetol 46:85-95

29. Rooney DP, Neely RD, Cullen C et al (1993) The effect of cortisol on glucose/glucose-6-phosphate cycle activity and insulin action. J Clin Endocrinol Metab 77:1180-1183

30. Vegiopoulos A, Herzig S (2007) Glucocorticoids, metabolism and metabolic diseases. Mol Cell Endocrinol 275:43-61

31. Giordano R, Picu A, Marinazzo E et al (2011) Metabolic and cardiovascular outcomes in patients with Cushing's syndrome of different aetiologies during active disease and 1 year after remission. Clin Endocrinol (Oxf) 75:354-360
32. American Diabetes Association (2012) Standards of medical care in diabetes-2012. Diabetes Care 35(Suppl 1):S11-S63

33. Munir A, Newell-Price J (2010) Management of diabetes mellitus in Cushing's syndrome. Neuroendocrinology 92(Suppl 1):82-85

34. Petersenn S, Schopohl J, Barkan A et al (2010) Pasireotide (SOM230) demonstrates efficacy and safety in patients with acromegaly: a randomized, multicenter, phase II trial. J Clin Endocrinol Metab 95:2781-2789

35. National Cancer Institute. Common Terminology Criteria for Adverse Events v3.0 (CTCAE), publish date: August 9, 2006. http://ctep.cancer.gov/protocolDevelopment/electronic_applications/docs/ctcaev3.pdf

36. Henry RR, Mudaliar S, Wetli-Hermosillo K, Ligueros-Saylan M, Chenji S, Golor G (2011) Mechanism and management of hyperglycemia associated with pasireotide: results from studies in healthy volunteers. In: 13th European Congress of Endocrinology, Rotterdam, the Netherlands (abstract P260)

37. Inzucchi SE, Bergenstal RM, Buse JB et al (2012) Management of hyperglycaemia in type 2 diabetes: a patient-centered approach. Position statement of the American Diabetes Association (ADA) and the European Association for the Study of Diabetes (EASD). Diabetologia 55:1577-1596

38. Shah P, Mudaliar S (2010) Pioglitazone: side effect and safety profile. Expert Opin Drug Saf 9:347-354

39. Esposito K, Chiodini P, Capuano A et al (2012) Basal supplementation of insulin lispro protamine suspension versus insulin glargine and detemir for type 2 diabetes: meta-analysis of randomized controlled trials. Diabetes Care 5:2698-2705

40. Reznik Y, Bertherat J, Borson-Chazot Fet al (2012) Management of hyperglycaemia in Cushing's disease: experts' proposals on the use of pasireotide. Diabetes Metab. doi:10.1016/j.diabet.2012.10.005

41. Tuomilehto J, Lindström J, Hellmich M et al (2010) Development and validation of a risk-score model for subjects with impaired glucose tolerance for the assessment of the risk of type 2 diabetes mellitus-the STOP-NIDDM risk-score. Diabetes Res Clin Pract 87:267-274

42. Lindström J, Tuomilehto J (2003) The diabetes risk score. A practical tool to predict type 2 diabetes risk. Diabetes Care 26:725-731 